\title{
Applying Dynamical Systems Theory to Optimize Libration Point Orbit Stationkeeping Maneuvers for WIND
}

\author{
Jonathan M. Brown ${ }^{*}$ and Jeremy D. Petersen ${ }^{\dagger}$ \\ a.i. solutions, Inc., Lanham, MD, 20706
}

\begin{abstract}
NASA's WIND mission has been operating in a large amplitude Lissajous orbit in the vicinity of the interior libration point of the Sun-Earth/Moon system since 2004. Regular stationkeeping maneuvers are required to maintain the orbit due to the instability around the collinear libration points. Historically these stationkeeping maneuvers have been performed by applying an incremental change in velocity, or $\Delta v$, along the spacecraft-Sun vector as projected into the ecliptic plane. Previous studies have shown that the magnitude of libration point stationkeeping maneuvers can be minimized by applying the $\Delta v$ in the direction of the local stable manifold found using dynamical systems theory. This paper presents the analysis of this new maneuver strategy which shows that the magnitude of stationkeeping maneuvers can be decreased by 5 to 25 percent, depending on the location in the orbit where the maneuver is performed. The implementation of the optimized maneuver method into operations is discussed and results are presented for the first two optimized stationkeeping maneuvers executed by WIND.
\end{abstract}

\section{Nomenclature}

$A(t)=$ partial derivative matrix of the CR3BP equations of motion with respect to the six element state

$b \quad=\quad \mathrm{y}$-intercept for a line

$\bar{D} \quad=$ vector from larger primary, $\mathrm{m}_{1}$, to the spacecraft, $\mathrm{m}_{3}$

$D F(X)=$ partial derivative matrix of the free variables, $\boldsymbol{X}$, with respect to the constraints, $\boldsymbol{F}(\boldsymbol{X})$

$d=$ scalar distance from the larger primary, $\mathrm{m}_{1}$, to the spacecraft, $\mathrm{m}_{3}$

$\bar{d} \quad=$ nondimensional vector from the larger primary, $\mathrm{m}_{1}$, to the spacecraft, $\mathrm{m}_{3}$

$D_{1} \quad=$ distance from $\mathrm{m}_{1}, \mathrm{~m}_{2}$ barycenter to larger primary, $\mathrm{m}_{1}$

$D_{2} \quad=$ distance from $\mathrm{m}_{1}, \mathrm{~m}_{2}$ barycenter to smaller primary, $\mathrm{m}_{2}$

$\boldsymbol{F}(\boldsymbol{X})=$ constraint equations for fixed-time multiple shooting algorithm

$\bar{f}=\quad=$ equations of motion for the spacecraft in the CR3BP

$\tilde{G} \quad=$ dimensional gravitational constant

$l^{*} \quad=$ characteristic length

$m^{*} \quad=$ characteristic mass

$m_{1} \quad=$ mass of the larger primary

$m_{2} \quad=$ mass of the smaller primary

$m_{3} \quad=$ mass of the spacecraft (zero)

$n=$ number of patch points

$\bar{p} \quad=$ dimensional position vector of $\mathrm{m}_{3}$ with respect to the system barycenter

$\bar{R} \quad=$ vector from smaller primary, $\mathrm{m}_{2}$, to the spacecraft, $\mathrm{m}_{3}$

$r=$ scalar distance from the smaller primary, $\mathrm{m}_{2}$, to the spacecraft, $\mathrm{m}_{3}$

$\bar{r} \quad=$ nondimensional vector from the smaller primary, $\mathrm{m}_{2}$, to the spacecraft, $\mathrm{m}_{3}$

$s=$ slope of a line

$t \quad=$ time

* Systems Engineer, Mission Services Division, 10001 Derekwood Ln. Ste. 215, Lanham, MD 20706, Member AIAA.

† Junior Systems Engineer, Mission Services Division, 10001 Derekwood Ln. Ste. 215, Lanham, MD 20706, Member AIAA. 


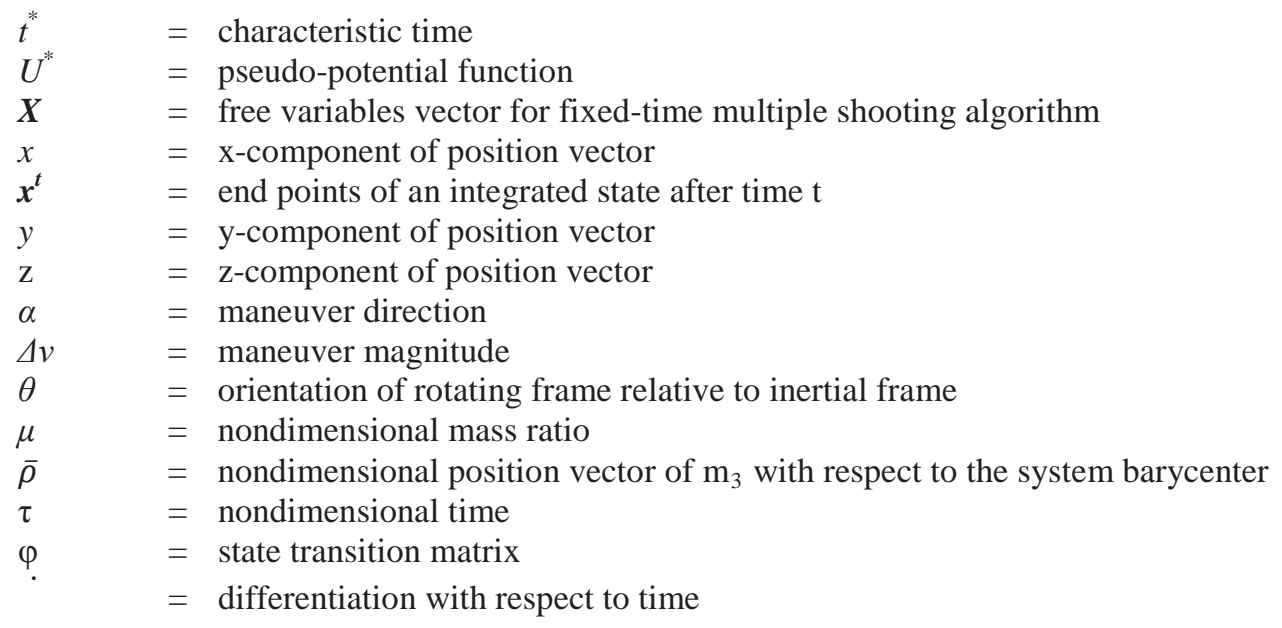

\section{Introduction}

$\mathrm{T}_{\mathrm{s}}^{\mathrm{H}}$

HE Global Geospace Science WIND satellite is a heliophysics mission operated by NASA at the Goddard Space Flight Center in Greenbelt, Maryland. It was launched in 1994 to improve understanding of the physics of solar terrestrial relations as a member of the International Solar Terrestrial Physics program ${ }^{\ddagger}$. After a decade spent in a series of exotic orbits, including 38 lunar swingbys and visits to the first and second libration points in the SunEarth/Moon system (L1 and L2), in 2004 WIND was placed into orbit around L1 where it has remained since.

Trajectories in the vicinity of the collinear libration points are inherently unstable, and thus periodic stationkeeping maneuvers are required to maintain the large amplitude Lissajous trajectory. The WIND mission refers to stationkeeping maneuvers as mid-course corrections (MCC), to remain consistent with terminology used throughout the mission. Since insertion into the Lissajous orbit in 2004, MCCs have been executed about every three months, or twice per revolution about L1. Because L1 is the location where the gravitational attraction between the Sun and the Earth/Moon barycenter (EMB) are equal, any error introduced by unmodeled perturbations will grow exponentially as one of the gravitational forces begins to dominate. Therefore MCCs are designed to counteract the effects of such perturbations, and the net change in velocity $(\Delta v)$ has historically been directed either toward or away from the Sun $^{1}$.

This maneuver design strategy has been intuitive, but as several previous studies have shown ${ }^{2-4}$, this is not the most efficient method for stationkeeping of libration point orbits (LPO) in terms of $\Delta \mathrm{v}$, and therefore fuel. This work will present the application of dynamical systems to compute the maneuver direction which minimizes $\Delta \mathrm{v}$ for any position in WIND's orbit, discuss the implementation of this new maneuver design process into operations, and show results for the first two MCCs which were performed using this methodology.

\section{Mission Description}

WIND has a cylindrical body, approximately 1.8 meters in height with a diameter of 2.4 meters. A series of booms and wires extend outward both radially and axially from the main body with sensors for some of the 8 science instruments onboard. The spacecraft is spin-stabilized about the $+\mathrm{Z}$ body axis, completing approximately 20 rotations per minute, and the spin axis is pointed within one degree of the direction of the South Ecliptic Pole. Figure 1 shows the spacecraft with the instrument locations denoted. Also depicted in Fig. 1 are the three different types of thrusters: axial, radial, and spin adjust. There are four of each type of thruster, for a total of 12 thrusters in all, as depicted in Fig. 2; all of the thrusters are draw from a blowdown hydrazine

\footnotetext{
${ }^{\ddagger}$ For more information, refer to the project website at $h t t p: / / w i n d . n a s a . g o v /$
} 

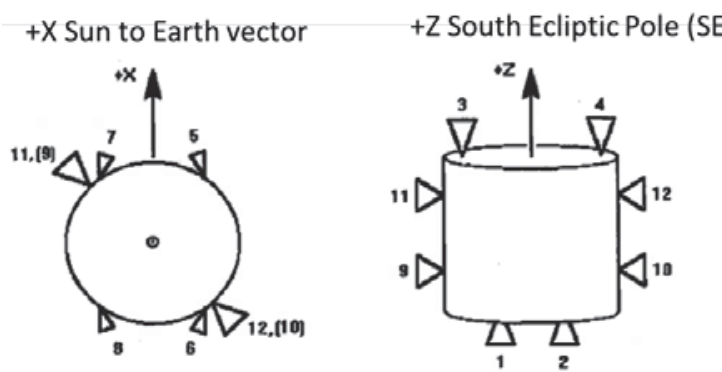

propulsion system. Given the spin axis orientation towards the South Ecliptic Pole and the fact that the instability in the vicinity of the libration points lies primarily in the ecliptic plane, the radial thrusters are used for LPO stationkeeping maneuvers. The radial thrusters are fired in pairs to achieve the desired $\Delta \mathrm{v}$, with each radial thuster providing 22 Newtons of force (beginning of life). Either thrusters 9 and 11 or thrusters 10 and 12 can be used to achieve the same net $\Delta \mathrm{v}$; however due to an anomaly with thruster 10, that pair is no longer used, leaving thrusters 9 and 11 to perform all radial $\Delta \mathrm{v}$ maneuvers.

After launching on November 1, 1994, WIND spent a Figure 2. WIND Thruster Diagram. Thrusters 1,2,3,4 are axial; thrusters 5,6,7,8 are spin control; and thrusters 9,10,11,12 are radial. decade traversing the Earth/Moon system. The trajectory during this time included several double lunar swingbys to control the line of apsides, lunar backflip transfers in order to reorient the line of apsides, a series of distant prograde orbits, and 38 targeted lunar flybys ${ }^{5-8}$. Following the final lunar flyby in late 2002, WIND was sent on a single loop around L1 and then briefly returned to the Earth/Moon system before being flung out to L2. The spacecraft again completed a single loop around the libration point, fell back into the Earth/Moon system, and was flung out to the opposite libration point, L1, where it arrived in mid-2004. This time however, orbit insertion and stationkeeping maneuvers were performed to maintain the libration point orbit, and the trajectory has remained in the L1 region for the last decade.

Several factors were considered when selecting the parameters for WIND's libration point orbit ${ }^{8}$. The Lissajous trajectory that was ultimately chosen allowed for minimum $\Delta \mathrm{v}$ expenditure at orbit insertion, while also maximizing the orbit lifetime before the $\mathrm{z}$ amplitude collapses and enters a 3 degree solar exclusion zone in the center of the orbit. Figure 3 shows a five year propagation of the current trajectory represented in the rotating libration point (RLP) frame. The $\mathrm{x}$-axis of this coordinate system points from the Sun to the EMB, the $\mathrm{z}$ axis is normal to the orbit plane of the EMB about the Sun, and the y-axis completes the right handed system.

\section{Legacy Maneuver Design Strategy}

Stationkeeping maneuvers are required for WIND approximately every three months due to the unstable nature of the collinear libration points. WIND does not have a reference trajectory, so the only requirement for maneuvering is to remain in orbit around L1 which greatly simplifies the maneuver planning process. A differential corrector is implemented to determine the $\Delta \mathrm{v}$ directed along the WIND-Sun vector necessary to minimize the $\mathrm{x}$ component of velocity at the fourth crossing of the $\mathrm{x}-\mathrm{z}$ plane in the rotating frame after the maneuver. An initial maneuver plan is calculated with the COTS software FreeFlyer ${ }^{\circledR}$ using an impulsive maneuver assuming the spin axis is aligned directly along the South Ecliptic Pole. As the maneuver date approaches, the Flight Operations Team (FOT) provides the most current estimate of the right ascension and declination of the spacecraft's spin axis. A new impulsive $\Delta \mathrm{v}$ is calculated based on the attitude update. 
The resultant impulsive $\Delta \mathrm{v}$ is input into the GOTS software Goddard General Maneuver Program, more commonly referred to as GMAN. This program implements a detailed attitude model for the spacecraft in order to generate the finite maneuver plan which achieves the desired $\Delta v$ while minimizing the applied torque to the spinstabilized attitude. The resultant maneuver plan contains the number of pulses that the radial thrusters must perform, the pulse width for each thruster, and the jet start angle for each thruster. The number of pulses indicates how many identical finite pulses are required to achieve the desired $\Delta \mathrm{v}$. The pulse width, measured in the spin plane, denotes the angle of rotation through which each thruster should fire per pulse. The jet start angle, also measured in the spin plane, is the angle where the pulse begins, and it is measured relative to the point where the sun sensor detects the Sun.

The pulse width for thruster 9 is fixed at 30 degrees, and GMAN computes the pulse width for thruster 11 which minimizes the resulting torque on the spin axis. The jet start angles for each thruster are both varied such that the net $\Delta \mathrm{v}$ is in the user specified direction while also minimizing attitude perturbations. The spacecraft is only capable of performing an integer number of these 30 degree pulses, so only discrete values of $\Delta v$ are achievable. In general, these possible maneuver magnitudes will differ from the desired impulsive $\Delta \mathrm{v}$ computed with FreeFlyer, so GMAN selects the number of pulses which minimizes the difference between the finite and impulsive $\Delta \mathrm{v}$ values. This residual $\Delta \mathrm{v}$ introduces an undesired velocity error into the orbit, and it can sometimes have a significant influence on the size of the following maneuver. Typical MCC magnitudes can vary between 5 and $50 \mathrm{~cm} / \mathrm{s}$; the $\Delta \mathrm{v}$ from each 30 degree pulse of the thrusters is about $0.75 \mathrm{~cm} / \mathrm{s}$. The number of pulses computed by GMAN can have an error of up to a half of a pulse, or $0.375 \mathrm{~cm} / \mathrm{s}$. For MCCs with relatively large magnitudes the performance of the propulsion system will tend to dominate the post-maneuver velocity residual, but for smaller maneuvers the difference between the discrete $\Delta \mathrm{v}$ and the desired $\Delta \mathrm{v}$ can have significant effect.

\section{Optimized Maneuver Strategy using Dynamical Systems Theory}

Previous studies have examined the optimization of stationkeeping for libration point orbits using dynamical systems theor $y^{2-4}$. These optimization studies have shown that the magnitude of the $\Delta \mathrm{v}$ can be minimized by maneuvering along the stable eigenvector of the state transition matrix, as expressed in the RLP frame, after propagating the state transition matrix for one full revolution around the libration point in the $\mathrm{x}$-y projection. The legacy maneuver design strategy for WIND does not take advantage of this potential $\Delta \mathrm{v}$ savings as the maneuver direction is always oriented along the projection of the spacecraft-Sun vector into the spacecraft spin plane.

\section{A. CR3BP Formulation}


The first step towards the implementation of an optimized maneuver strategy using dynamical systems theory begins with a semi-analytical study using the well-established circular restricted three body problem (CR3BP) model $^{9}$. The CR3BP model, shown in Fig. 4 , is a rotating frame composed of three bodies, two primaries, $m_{1}$ and $m_{2}$, and an infinitesimally small third body, $m_{3}$. For this application, $m_{1}$ is the Sun, $m_{2}$ is the Earth/Moon system, and $m_{3}$ is the WIND spacecraft. The two primaries orbit about their shared barycenter at a constant angular rate. The $\mathrm{x}$-axis of the frame is defined as the line passing through the two primaries, the $\mathrm{z}$-axis is perpendicular to the plane of rotation, and the y-axis completes the right-handed triad. It is often useful to nondimensionalize the equations of motion based on a set of characteristic quantities. The characteristic values for length $\left(l^{*}\right)$, mass $\left(m^{*}\right)$, and time $\left(t^{*}\right)$, are defined as

$$
l^{*}=D_{1}+D_{2}, \quad m^{*}=m_{1}+m_{2}, \quad t^{*}=\sqrt{\frac{l^{* 3}}{\tilde{G} m^{*}}}
$$

where $D_{1}$ and $D_{2}$ are the distances between the primaries and their barycenter, $\mathrm{m} 1$ and $\mathrm{m} 2$ are the masses of the primaries, and $\tilde{G}$ is the dimensional gravitational constant.

Using the characteristic quantities defined in Eqs. (1), the nondimensional time parameter, $\tau$, and mass parameter, $\mu$, can be defined as

$$
\tau=\frac{t}{t^{* \prime}} \quad \mu=\frac{m_{2}}{m^{*}}
$$

and the nondimensional vectors describing the position of the spacecraft relative to the two primaries, $\bar{r}$ and $\bar{d}$, and relative to the barycenter of the system, $\bar{\rho}$, are defined as

$$
\bar{r}=\frac{\bar{R}}{l^{*}}, \quad \bar{d}=\frac{\bar{D}}{l^{*}}, \quad \bar{\rho}=\frac{\bar{p}}{l^{*}}=x \hat{x}+y \hat{y}+z \hat{z} .
$$

The unit vectors $\hat{x}, \hat{y}$, and $\hat{z}$

With the nondimensional quantities established, the equations of motion for the third body in the system can be written as follows:

$$
\ddot{x}-2 \dot{y}=\frac{\partial U^{*}}{\partial x}, \quad \ddot{y}+2 \dot{x}=\frac{\partial U^{*}}{\partial y}, \quad \ddot{z}=\frac{\partial U^{*}}{\partial z},
$$

where the dots represent differentiation with respect to nondimensional time. $U^{*}$ is the pseudo-potential function,

$$
U^{*}=\frac{1-\mu}{d}+\frac{\mu}{r}+\frac{1}{2}\left(x^{2}+y^{2}\right)
$$

where $d$ and $r$ are scalar distances,

$$
d=\sqrt{(x+\mu)^{2}+y^{2}+z^{2}}, \quad r=\sqrt{(x-1+\mu)^{2}+y^{2}+z^{2}} .
$$

The optimal maneuver direction is located along the stable eigenvector of the monodromy matrix ${ }^{10}$; to calculate this matrix, the state transition matrix, $\varphi$, associated with the CR3BP equations of motion, Eqs. (2), must be numerically integrated for one period about the libration point. The first order differential equation governing the state transition matrix is 


$$
\dot{\varphi}\left(t, t_{o}\right)=A(t) \varphi\left(t, t_{o}\right)
$$

where $A(t)$ is the Jacobian matrix that is composed of the partial derivatives of the equations of motion, $\bar{f}$, with respect to each of the six states, $\bar{x}$,

$$
\begin{gathered}
A(t)=\frac{\partial \bar{f}}{\partial \bar{x}}=\left[\begin{array}{cccccc}
0 & 0 & 0 & 1 & 0 & 0 \\
0 & 0 & 0 & 0 & 1 & 0 \\
0 & 0 & 0 & 0 & 0 & 1 \\
U_{x x}^{*} & U_{x y}^{*} & U_{x z}^{*} & 0 & 2 & 0 \\
U_{y x}^{*} & U_{y y}^{*} & U_{y z}^{*} & -2 & 0 & 0 \\
U_{z x}^{*} & U_{z y}^{*} & U_{z z}^{*} & 0 & 0 & 0
\end{array}\right] \\
U_{i j}^{*}=\frac{\partial U^{*}}{\partial i \partial j}
\end{gathered}
$$

\section{B. Creating a Reference LPO}

In order to create a reference trajectory in the CR3BP model necessary to generate the eigenvectors, a single revolution of the orbit from the operational stationkeeping software, FreeFlyer, was generated and discretized into multiple patch points. The patch points were nondimensionalized and imported into MATLAB ${ }^{\circledR}$. A fixed-time multiple shooting algorithm is implemented using the CR3BP model in MATLAB. A detailed overview of differential correcting algorithms can be found in Ref. 9. Below is a brief overview and description of the iterative fixed-time multiple shooting algorithm implemented for this investigation.

The multiple shooting algorithm is formulated as a series of constraints and free variables. The reference trajectory is discretized into a series of patch points that are stacked to generate a vector of length $n$ free variables,

$$
X=\left[\begin{array}{c}
x_{1} \\
\vdots \\
x_{n}
\end{array}\right] .
$$

The vector $\boldsymbol{X}$ is $6 n \times 1$ in size, where each element $\boldsymbol{x}_{\boldsymbol{i}}$ is a six element position and velocity state. For a fixed-time algorithm, each patch point has an associated integration time. The patch points are integrated simultaneously for their fixed integration time. This integration creates a series of segments that are subjected to a series of constraints. For trajectory design, the constraints $\mathbf{F}(\mathbf{X})$, a vector of length $6(n-1)$, are used to establish position and velocity continuity between the initial and end points of the integrated segments,

$$
F(X)=\left[\begin{array}{c}
x_{2}^{t}-x_{2} \\
\vdots \\
x_{n}^{t}-x_{n}
\end{array}\right]=0
$$

After propagation, the constraint equation is checked to determine if a desirable continuation tolerance has been met. Initially, there will be discontinuities between the integration segments. An update equation is necessary to determine the modifications necessary to the free variables for the next step in the iteration process. For this algorithm, a minimum norm solution is used

$$
\boldsymbol{X}^{\boldsymbol{j}+\boldsymbol{1}}=\boldsymbol{X}^{\boldsymbol{j}}-D \boldsymbol{F}\left(\boldsymbol{X}^{\boldsymbol{j}}\right)^{T}\left[D \boldsymbol{F}\left(\boldsymbol{X}^{j}\right) D \boldsymbol{F}\left(\boldsymbol{X}^{\boldsymbol{j}}\right)^{T}\right]^{-1} \boldsymbol{F}\left(\boldsymbol{X}^{\boldsymbol{j}}\right),
$$

where $\boldsymbol{D F}\left(\boldsymbol{X}^{\boldsymbol{j}}\right)$ is the Jacobian matrix that relates the partial derivatives of the constraints with respect to the free variables. The state transition matrix can be used to evaluate the partial derivatives of the $6(n-1) \times 6 n$ Jacobian matrix numerically. Blank entries denote a $6 x 6$ sub matrix of zeroes. 


$$
D \boldsymbol{F}(\boldsymbol{X})=\frac{\partial \boldsymbol{F}(\boldsymbol{X})}{\partial \boldsymbol{X}}=\left[\begin{array}{cccc}
\varphi\left(t_{2}, t_{1}\right) & -I_{6 x 6} & & \\
& \ddots & \ddots & \\
& & \varphi\left(t_{n}, t_{n-1}\right) & -I_{6 x 6}
\end{array}\right]
$$

The update equation sets the initial conditions for the next iteration. The sequence continues until the constraint equation satisfies a tolerance near zero (for example 1E-12). The reference orbit in the CR3BP problem is established once the continuity constraints have been satisfied.

\section{Determining Local Stability}

The reference orbit is then decomposed into multiple discrete points. Each of these points, along with the state transition matrix, is propagated for a single period in order to generate the monodromy matrix. Each discrete point from the reference trajectory now has an associated monodromy matrix and, therefore, a set of eigenvalues and eigenvectors that describes the stability characteristics of that point. Stability analysis involving the monodromy matrix uses 1 as the boundary; eigenvalues with magnitudes greater than 1 are unstable while magnitudes less than 1 are stable $^{11}$. For this system, we are only concerned with the two eigenvalues that have no imaginary component, one stable and one unstable. The optimal maneuver direction is found by calculating the angle generated by the $\mathrm{x}$ and y-component of the eigenvector corresponding to the stable eigenvalue. The z-component of the eigenvector is neglected because the net $\Delta \mathrm{v}$ from stationkeeping maneuvers using WIND's radial thrusters is constrained to the spin plane, which is assumed to be parallel to the ecliptic plane since the spin axis is pointing toward the south ecliptic pole.

In order to organize the optimal maneuver directions, each discrete point in the reference orbit is classified by the angle between the position projection in the $x-y$ plane and the +x-axis, measured counter clockwise. This classification creates a unique orbit angle, or maneuver location, for each discrete point in the reference trajectory. Figures 5 and 6 show two representations of the eigenvectors: Fig. 5 shows the vectors at various locations throughout the orbit, and Fig. 6 contains the angle created by the $\mathrm{x}$ - and $\mathrm{y}$ - components of the stable and unstable eigenvector as a function of the orbit angle in the $\mathrm{x}$-y plane. The z-components of the eigenvectors are neglected as they are significantly smaller than the $\mathrm{x}$ - and $\mathrm{y}$-components, and because we are only considering maneuvers in WIND's spin plane, which is parallel to the ecliptic plane. In both figures, the red lines correspond to the unstable eigenvector while the blue lines correspond to the stable eigenvector and optimal maneuver direction. 


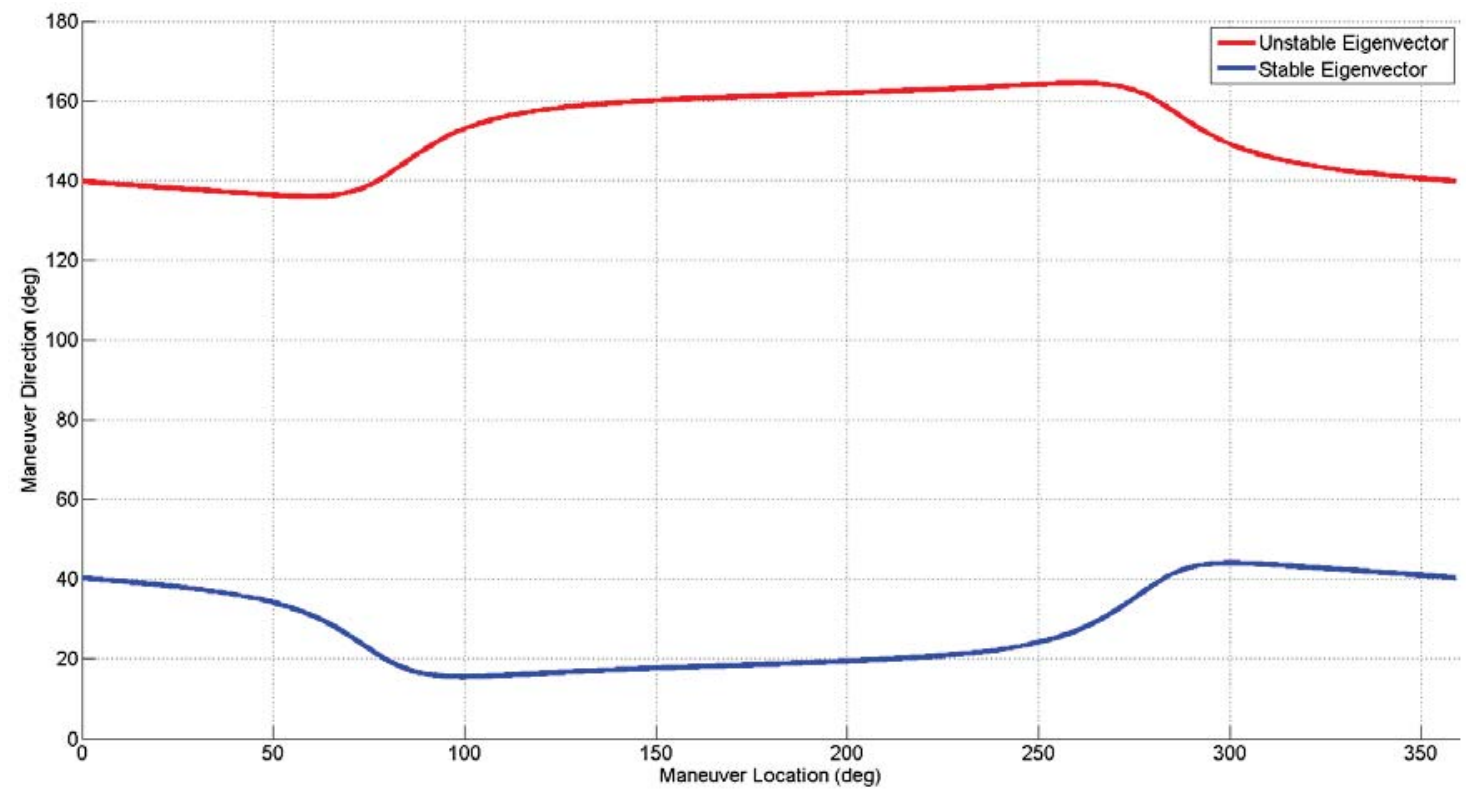

Figure 6. Stable and Unstable Eigenvectors. Directions of the eigenvectors vary throughout the orbit. Both angles are measured relative to the $R L P+x$-axis.

\section{Validation Using a Full Ephemeris Model}

A simulation was conducted in FreeFlyer in order to validate the results derived using dynamical systems theory. A ray scan was performed by varying the $\Delta \mathrm{v}$ over the entire quadrant of the spin phase which contains the stable eigenvector, from the $+\mathrm{x}$-axis to the $+\mathrm{y}$-axis. The $\Delta \mathrm{v}$ was varied at 1 degree intervals, and for each possible maneuver direction, the $\Delta \mathrm{v}$ magnitude was computed using the operational differential correction algorithm to determine the maneuver size required achieve a perpendicular crossing after four $\mathrm{X}-\mathrm{z}$ plane crossings. Maneuvers which resulted in a negative $\Delta v$ corresponded to solutions in the third quadrant rather than the first. Figure 6 shows the results for one of the maneuvers that was examined, MCC-47. The angle in Fig. 7 represents the direction of the $\Delta \mathrm{v}$ relative to the RLP $+\mathrm{x}$-axis as rotated about the RLP +z-axis, measured in degrees. The $\Delta \mathrm{v}$ magnitude is measured radially in units of meters per second, and the blue line shows the variation in maneuver size as the direction of the $\Delta v$ is rotated in the ecliptic plane. The red vector shows the maneuver which was actually executed, and it was computed using the legacy maneuver scheme. The blue vector represents the minimum $\Delta v$ solution, and the blue line represents the magnitudes computed for each of the directions 
analyzed.

It is interesting to note that the possible solutions form a straight line in this representation. The ray scan was repeated for every MCC from January 2012 through January 2014, a total of 10 maneuvers, and it was found that each of the data sets exactly fit a straight line. This implies that regardless of maneuver direction, the component of the $\Delta \mathrm{v}$ in the minimum direction is required to maintain the orbit. This behavior will be revisited in order to find the maneuver direction where the impulsive and finite $\Delta v$ magnitudes are equal.

Table 1 shows the results for each of these maneuvers. The legacy $\Delta \mathrm{v}$ is the maneuver magnitude that was actually performed, and it was computed using the method described previously. The optimized $\Delta \mathrm{v}$ is the minimum $\Delta \mathrm{v}$ found from the ray scan, and the angle from RLP $+\mathrm{x}$ is the direction where the minimum $\Delta \mathrm{v}$ was found. The column "Difference from DST" shows the difference between the angle found in the previous column, and the angle computed for that maneuver location using dynamical systems theory. Finally, the savings shown in the last column is the relative decrease in $\Delta \mathrm{v}$ that could have been achieved by directing the maneuver along the stable eigenvector. The minimum for each of the 10 maneuvers was found to be within 1 degree of the expected value, and this margin of error was expected given the 1 degree resolution of the ray scan. Depending on the location of the maneuver in the libration point orbit, the magnitude of the MCCs can be decreased by 5 to 25 percent. This variation in efficiency is primarily the result of the angle between the stable eigenvector and the WIND-Sun vector.

\section{Operational Implementation}

\section{A. Computation of the Stable Eigenvector}

After observing strong agreement between the semi-analytical study using the CR3BP model and a full ephemeris model in FreeFlyer, the maneuver planning team implemented this optimized strategy into the stationkeeping planning process. A polynomial curve fit of the optimal maneuver direction as a function of maneuver location in the x-y plane, shown in Figure 6, was generated and added to the impulsive maneuver planning script. At the maneuver epoch, the spacecraft location in the ecliptic plane is found and input into the polynomial curve fit. The direction of the impulsive $\Delta \mathrm{v}$ is adjusted based on the value obtained from the polynomial. Once the maneuver direction is established, the maneuver planning process continues as usual. The impulsive $\Delta v$ from the FreeFlyer script is input into GMAN and a finite maneuver plan containing the number of finite pulses, the pulse width, and the jet start angle is generated.

Because the maneuver direction is being varied within the spin plane, the modifications to the maneuver planning process are effectively transparent to the FOT. Two of the three parameters delivered as part of the finite maneuver plan change: the number of finite pulses will decrease due to the efficiency gain, and the jet start angles will also decrease because the maneuver direction is earlier in the spin phase than the Sun-spacecraft vector. Neither of these changes have any impact on the sequence of commands sent to the spacecraft during a maneuver, only the values are changed. In fact, these parameters are varied slightly for all maneuvers even using the legacy maneuver planning strategy; the magnitude of the burns is of course different each time, and the jet start angles vary slightly 
for different locations in the orbit as the WIND-Sun vector changes. The fact that WIND's spin axis is aligned with the south ecliptic pole is a strong reason why this maneuver optimization strategy was straight forward to implement. The ability to change the maneuver direction in the $x-y$ plane in order to match the optimized direction along the stable eigenvector is a simple alteration to the jet start angle such that each pulse is centered about the stable eigenvector instead of the spacecraft-Sun vector.

\section{B. Adjustment of the Jet Start Angles to Minimize Residual $\Delta \mathrm{v}$}

With the maneuver optimization implemented in the FreeFlyer script used to compute the impulsive $\Delta v$, the corresponding finite maneuver plan can be generated in GMAN by simply inputting the optimized impulsive $\Delta \mathrm{v}$ vector. As discussed previously, the finite maneuver plan results in a discrete $\Delta \mathrm{v}$ based on the number of fixed-width pulses. In general, the $\Delta \mathrm{v}$ output from the finite maneuver plan contains a residual $\Delta \mathrm{v}$ when compared against the impulsive $\Delta \mathrm{v}$. This residual is equivalent to as much as a half of a pulse in either direction. If the overall maneuver size is large this error can be insignificant, however, for very small maneuvers it can represent a significant percentage of the total maneuver.

The maneuver error, or residual $\Delta \mathrm{v}$, defines the following station keeping maneuver. As a quick approximation technique derived from operational experience, the maneuver magnitude for the next stationkeeping maneuver is the residual $\Delta \mathrm{v}$ from the previous maneuver and is doubled every 16 days ${ }^{1}$. As a simple example, a stationkeeping maneuver with $1 \mathrm{~cm} / \mathrm{s}$ residual $\Delta \mathrm{v}$ error will require a $2 \mathrm{~cm} / \mathrm{s}$ maneuver if performed 16 days later, a $4 \mathrm{~cm} / \mathrm{s}$ maneuver if performance 32 days later, and so on.

Using the legacy maneuver planning process the residual error resulting from the difference between the impulsive model and finite model was simply accepted as a limitation of the system. By using the tools developed to optimize the maneuver direction it is now possible to eliminate residual $\Delta \mathrm{v}$ by adjusting the jet start angle away from the optimal direction such that the $\Delta \mathrm{v}$ from the impulsive maneuver plan matches the $\Delta \mathrm{v}$ from the finite maneuver plan generated in GMAN. Any remaining residual $\Delta \mathrm{v}$ will then be purely a function of the propulsion system performance, and not a bias in the expected $\Delta \mathrm{v}$.

Figure 8 shows an example of this process. The blue line is the $\Delta \mathrm{v}$ as a function of maneuver direction, and the blue vector is the optimized $\Delta v$ computed in FreeFlyer. The green vector that extends just beyond the blue vector is the finite $\Delta \mathrm{v}$ computed in GMAN, and the dashed green arc shows that maneuver magnitude swept through the entire quadrant. To eliminate any residual $\Delta \mathrm{v}$ that results from the discrete number of pulses, the maneuver direction must be adjusted to one of the two points where the blue line and the green arc intersect. The yellow and magenta vectors represent the two maneuvers in the directions of these solutions, and they are equidistant from the optimized impulsive $\Delta \mathrm{v}$.

The ray scan is computationally intensive, so it is desirable to solve for the relationship between maneuver direction and magnitude using an analytical technique if possible. The linearity in the $\Delta \mathrm{v}$ as a function of maneuver direction noted previously makes this possible. By definition, the vector from the origin to the minimum $\Delta \mathrm{v}$ location will be perpendicular to the line of data points generated by the ray scan. Once the direction and magnitude of the minimum $\Delta \mathrm{v}$ have been found using the impulsive targeting script, the relationship between the maneuver direction and magnitude can be found by solving for the line that is perpendicular to the minimum $\Delta \mathrm{v}$ vector.

Denoting the direction and magnitude of the minimized $\Delta \mathrm{v}$ as $\theta_{\min }$ and $\Delta v_{\min }$, respectively, these values can be converted to Cartesian coordinates to find $x_{\min }$ and $y_{\min }$. The slope of the line, $s$, perpendicular to the minimum solution vector is the negative inverse of the slope of the vector from the origin to the point $\left(x_{\min }, y_{\min }\right)$. The $\mathrm{y}$ intercept, $b$, can then be found using the standard equation for a Cartesian line. Converting the line back to polar coordinates results in the following relationship between the maneuver direction, $\alpha$, and the maneuver magnitude, $\Delta v:$ 


$$
\alpha=\sin ^{-1}\left(s \cos \alpha+\frac{b}{\Delta v}\right)
$$

This transcendental equation is then solved iteratively using Newton's method, where $\Delta v$ is the magnitude of the MCC generated from the finite maneuver plan in GMAN.

The above process assumes that the GMAN finite $\Delta v$ is larger than the impulsive $\Delta v$ from FreeFlyer. If the finite $\Delta \mathrm{v}$ is smaller than the impulsive value then the above method will not work; obviously it is not possible to adjust the angle to achieve smaller $\Delta \mathrm{v}$ than the already-computed minimum. To put it another way, the green arc in Fig. 7 will lie entirely inside the blue line, and the two curves never intersect. In this situation, the impulsive $\Delta \mathrm{v}$ which is input to GMAN must be increased slightly. Each pulse imparts a $\Delta v$ of roughly $0.75 \mathrm{~cm} / \mathrm{s}$, so by increasing the magnitude by that value, the resulting finite plan will have an additional thruster pulse. This results in a finite $\Delta \mathrm{v}$ larger than the impulsive $\Delta v$, and then it is possible to find and adjust the maneuver direction in order to eliminate the residual $\Delta \mathrm{v}$.

\section{Results}

The new maneuver planning process of optimizing the $\Delta v$ and then adjusting the maneuver direction to eliminate the undesirable residual $\Delta v$ has been applied successfully to two maneuvers, MCC-51 and MCC-52. MCC-51 was executed on April 10, 2014, and Table 2 shows the results of maneuver planning for the three different methods.

The optimized method for MCC-51 reduced the impulsive $\Delta \mathrm{v}$ required by $5.59 \%$ by changing the direction by 19.260 degrees. Using the finite burn modeling, the $\Delta v$ savings decreased slightly to $4.96 \%$ due to the discrete resolution provided by the integer number of pulses. However, this finite plan resulted in a residual $\Delta \mathrm{v}$ of 0.5335 $\mathrm{cm} / \mathrm{s}$, or $1.16 \%$ higher than required. To counteract this, the jet start angles were adjusted by 8.64 degrees relative to the optimized maneuver direction. This resulted in an offset of 10.62 degrees from the sun-pointing method, and increased the required impulsive $\Delta v$ slightly to match the finite $\Delta v$ from GMAN to within $0.0038 \mathrm{~cm} / \mathrm{s}$, or $0.01 \%$.

The actual $\Delta v$ observed during MCC-51 was $45.4815 \mathrm{~cm} / \mathrm{s}$, or $2.61 \%$ less than the predicted finite value, which is well within the range of past maneuver performance. The total velocity error in the orbit immediately after the maneuver is the difference between the observed $\Delta v$ and the impulsive $\Delta v$ for the adjusted maneuver direction, which was $1.2192 \mathrm{~cm} / \mathrm{s}$ for this maneuver. This velocity error grows exponentially, and the magnitude of the next maneuver can be quickly estimating by assuming that cost to correct the error doubles every 16 days. MCC-52 was scheduled for June 10, 2014, so knowing that the residual would double roughly 3.8 times, the $\Delta \mathrm{v}$ was estimated to be roughly $17 \mathrm{~cm} / \mathrm{s}$. This was confirmed when impulsive and finite maneuver planning for MCC-52 yielded the results shown in Table 3.

Table 3. MCC-52 Maneuver Plan

\begin{tabular}{|l|c|c|c|}
\hline & Sun-Pointing Method & Optimized $\Delta \mathbf{v}$ & Adjusted Pulses \\
\hline Maneuver Location (deg) & 55.0637 & 55.0637 & 55.0637 \\
\hline Maneuver Direction (deg) & 0.3597 & 23.5043 & 14.8531 \\
\hline Impulsive $\Delta v(\mathrm{~cm} / \mathrm{s})$ & 17.1503 & 15.7446 & 15.9258 \\
\hline Pulses & 25 & 23 & 23 \\
\hline Jet Start Angles (deg) & $99.240 / 101.816$ & $75.960 / 78.536$ & $84.600 / 87.176$ \\
\hline Discrete $\Delta$ v from GMAN (cm/s) & 17.4208 & 15.9258 & 15.9258 \\
\hline
\end{tabular}

11

American Institute of Aeronautics and Astronautics 
The impulsive $\Delta \mathrm{v}$ computed using the legacy Sun-pointing method was very near the estimate of $17 \mathrm{~cm} / \mathrm{s}$, and the optimized impulsive $\Delta \mathrm{v}$ decreased the magnitude by $8.19 \%$ at an angle of 23.145 degrees. The finite maneuver plan again resulted in a slightly larger $\Delta \mathrm{v}$ than required, so the angle was adjusted by 8.65 degrees to eliminate the residual $\Delta \mathrm{v}$.

\section{Conclusion}

Results from previous studies which showed that stationkeeping of libration point orbits can be optimized by maneuvering along the stable eigenvector of the state transition matrix were successfully applied to operations for the WIND mission. Analysis of over two years of past MCC maneuvers found that the $\Delta \mathrm{v}$ cost can be decreased by 5 to 25 percent, depending on the location in the orbit where the MCC is performed. As a result of WIND's spin axis orientation, the maneuver direction can easily be varied by changing the phase in the spin period when the radial thrusters are pulsed. However, maneuvers using the radial thrusters must perform a whole number of fixed-width pulses, so in general the exact minimum $\Delta \mathrm{v}$ cannot be achieved, resulting in some undesirable residual velocity. The linear variation of the $\Delta \mathrm{v}$ magnitude as a function of maneuver direction in the spin plane was used to develop a technique which eliminates the residual $\Delta \mathrm{v}$. The maneuver direction is adjusted away from the stable eigenvector until the finite $\Delta \mathrm{v}$ exactly matches the $\Delta \mathrm{v}$ required to maintain the orbit. This method of optimizing the $\Delta \mathrm{v}$ and then adjusting the maneuver direction to eliminate the residual velocity has been successfully applied to two MCCs, reducing the $\Delta \mathrm{v}$ by $4.96 \%$ and $8.58 \%$ relative to the legacy maneuver design method.

\section{Acknowledgments}

This work was conducted under NASA contracts NNG10CP02C and NNG14CR57C.

\section{References}

${ }^{1}$ Roberts, C. E., "Long Term Missions at the Sun-Earth Libration Point L1: ACE, SOHO and WIND,” AAS 11-485, AAS/AIAA Astrodynamics Specialist Conference, Girdwood, Alaska, August, 2011.

${ }^{2}$ Folta, D. C., Pavlak, T. A., Howell, K. C., Woodard, M. A., and Woodfork, D. M., "Stationkeeping of Lissajous Trajectories in the Earth-Moon System with Applications to ARTEMIS," $20^{\text {th }}$ AAS/AIAA Space Flight Mechanics Meeting, San Diego, CA, February, 2010.

${ }^{3}$ Pavlak, T. A., and Howell, K. C., "Strategy for Long-Term Libration Point Stationkeeping in the Earth-Moon System," AAS/AIAA Astrodynamics Specialist Conference, Girdwood, AK, August, 2011.

${ }^{4}$ Folta, D., Woodard, M., Pavlak, T., Haapala, A., and Howell, K., "Earth-Moon Libration Stationkeeping: Theory, Modeling, and Operations," Acta Astronautica. Vol. 94, No. 1, January-February 2014, Pages 421-433, ISSN 0094-5765.

${ }^{5}$ Franz, H., Sharer, P., Ogilvie, K., and Desch, M., "WIND Nominal Mission Performance and Extended Mission Design," The Journal of the Astronautical Sciences. Vol. 49, No. 1, January-March 2001, pp. 145-167.

${ }^{6}$ Franz, H., "WIND Lunar Backflip and Distant Prograde Orbit Implementation,” AAS 01-173, AAS/AIAA

Space Flight Mechanics Meeting, Santa Barbara, California, February, 2001.

${ }^{7}$ Franz, H., "Design of Earth Return Orbits for the WIND Mission,” AAS 02-170, AAS/AIAA Space Flight Mechanics Meeting, San Antonio, Texas, January, 2002.

${ }^{8}$ Franz, H., “A WIND Trajectory Design Incorporating Multitple Transfers Between Libration Points,” AIAA-2002-4525, AIAA /AAS Astrodynamics Specialist Conference, Monterey, California, August, 2002.

${ }^{9}$ Pavlak, T. A., "Mission Design Applications in the Earth-Moon System: Transfer Trajectories and Stationkeeping," Master's Thesis, Aeronautics and Astronautics Dept., Purdue Univ., West Lafayette, IN, 2010.

${ }^{10}$ Grebow, D. J., "Trajectory Design in the Earth-Moon System and Lunar South Pole Coverage," Ph.D. Dissertation, Aeronautics and Astronautics Dept., Purdue Univ., West Lafayette, IN, 2010.

${ }^{11}$ Geisel, C. D., "Spacecraft Orbit Design in the Cricular restricted Three-Body Problem Using Higher-Dimensional Poincare Maps,” Ph.D. Dissertation, Aeronautics and Astronautics Dept., Purdue Univ., West Lafayette, IN, 2013. 